\title{
Runs of homozygosity and distribution of functional variants in the cattle genome
}

\author{
Qianqian Zhang ${ }^{1,2^{*}}$, Bernt Guldbrandtsen ${ }^{1}$, Mirte Bosse ${ }^{2}$, Mogens S Lund ${ }^{1}$ and Goutam Sahana ${ }^{1 *}$
}

\begin{abstract}
Background: Recent developments in sequencing technology have facilitated widespread investigations of genomic variants, including continuous stretches of homozygous genomic regions. For cattle, a large proportion of these runs of homozygosity $(\mathrm{ROH})$ are likely the result of inbreeding due to the accumulation of elite alleles from long-term selective breeding programs. In the present study, $\mathrm{ROH}$ were characterized in four cattle breeds with whole genome sequence data and the distribution of predicted functional variants was detected in $\mathrm{ROH}$ regions and across different ROH length classes.

Results: On average, $19.5 \%$ of the genome was located in $\mathrm{ROH}$ across four cattle breeds. There were an average of $715.5 \mathrm{ROH}$ per genome with an average size of $\sim 750 \mathrm{kbp}$, ranging from 10 (minimum size considered) to 49,290 $\mathrm{kbp}$. There was a significant correlation between shared short $\mathrm{ROH}$ regions and regions putatively under selection $(p<0.001)$. By investigating the relationship between $\mathrm{ROH}$ and the predicted deleterious and non-deleterious variants, we gained insight into the distribution of functional variation in inbred $(\mathrm{ROH})$ regions. Predicted deleterious variants were more enriched in $\mathrm{ROH}$ regions than predicted non-deleterious variants, which is consistent with observations in the human genome. We also found that increased enrichment of deleterious variants was significantly higher in short ( $<100 \mathrm{kbp}$ ) and medium (0.1 to $3 \mathrm{Mbp}$ ) ROH regions compared with long ( $>3 \mathrm{Mbp}) \mathrm{ROH}$ regions $(P<0.001)$, which is different than what has been observed in the human genome.

Conclusions: This study illustrates the distribution of $\mathrm{ROH}$ and functional variants within $\mathrm{ROH}$ in cattle populations. These patterns are different from those in the human genome but consistent with the natural history of cattle populations, which is confirmed by the significant correlation between shared short $\mathrm{ROH}$ regions and regions putatively under selection. These findings contribute to understanding the effects of inbreeding and probably selection in shaping the distribution of functional variants in the cattle genome.
\end{abstract}

Keywords: Runs of homozygosity, Polymorphisms, Inbreeding, Cattle, Genome sequencing

\section{Background}

Dairy cattle have been subjected to more than 60 years of intense selection for traits to enhance milk production [1-4]. Relatively few bulls were chosen to produce thousands of daughters, resulting in large half-sib families. Traditionally, cattle breeders estimate the inbreeding coefficient by the degree of parental relatedness using pedigree and genotype data [5-8]. The rate of inbreeding in cattle populations has increased in recent years $[6,9$, $10]$, and there is a strong correlation between inbreeding levels and reduced fitness [11]. This can be explained by

\footnotetext{
* Correspondence: Qianqian.zhang@mbg.au.dk; Goutam.sahana@mbg.au.dk 'Department of Molecular Biology and Genetics, Center for Quantitative Genetics and Genomics, Aarhus University, Tjele DK-8830, Denmark Full list of author information is available at the end of the article
}

the increased risk of homozygosity for deleterious alleles as inbreeding increases [12]. Thus, high levels of inbreeding in populations will result in inbreeding depression $[10,13]$. Reduced variability also leads to a reduced selection response in breeding programs [14]. Thus, maintaining genetic diversity is crucial in cattle breeding populations.

The availability of high-throughput whole genome sequencing for substantial numbers of animals has opened new avenues in examining genetic diversity and led to reliable and detailed investigation of large chromosome segments, including stretches of homozygous genomic regions [15]. Runs of homozygosity ( $\mathrm{ROH})$ are contiguous homozygous stretches in an individual genome due to transmission of identical haplotypes from parents to 
offspring. $\mathrm{ROH}$ detection can be used to improve mating systems and minimize inbreeding. However, the effects of inbreeding vary among individuals and populations, and it has long been of interest to explore the mechanisms of inbreeding depression and deleterious variants at the genomic level [16-18]. Recently, $\mathrm{ROH}$ extracted from SNP chip data have been used to study the population history of different cattle breeds [19]. Purfield et al. [19] claimed that both natural and artificial selection of cattle, as well as demographic processes, have resulted in breeds with extensive phenotype variation. $\mathrm{ROH}$ may provide useful information on how these processes work in disparate populations, especially for cattle due to recent intense selection of sires, artificial insemination, and embryo transfer in some cattle breeds [19]. However, ROH estimated from SNP chip data may miss short and medium $\mathrm{ROH}$ due to limited resolution. Additionally, SNP arrays suffer from ascertainment biases due to inclusion of SNPs with high minor allele frequencies [20].

Consanguineous mating, population size reduction, and selection result in long homozygous regions along the genome $[15,16]$. Two copies of a genome segment in an individual inherited from a common ancestor without recombination are identical-by-descent (IBD). $\mathrm{ROH}$ that arise due to inbreeding tend to be fairly long and are dispersed more or less randomly throughout the genome. Accumulation of deleterious variants by definition creates fitness consequences, particularly when homozygous $[20$, 21]. Distribution of functional homozygote variants provides information on enrichment of deleterious homozygotes within homozygous regions compared with neutral homozygotes. Charlesworth et al. [22] suggested that a large number of weakly deleterious variants are purged by negative selection. A human genome study [23] indicated that purifying selection interacted with founder effects during demographic processes, affecting the proportion of recessive deleterious variants. Recently, based on variant annotations, Szpiech et al. [24] reported long $\mathrm{ROH}$ enriched deleterious variation in the human genome. By counting deleterious and neutral variants inside and outside $\mathrm{ROH}$ in humans, they determined that out of the two competing hypotheses regarding patterns of deleterious variation in the human genome - namely, whether a smaller or larger proportion of all deleterious homozygotes resided in $\mathrm{ROH}$ regions compared with neutral homozygotes [24], a larger proportion of deleterious homozygote variants were found, and deleterious variants in long $\mathrm{ROH}$ accumulated more rapidly compared to short and medium $\mathrm{ROH}$ in the human genome.

Cattle domestication was initiated approximately 10,000 years ago, with evidence of at least two separate domestication events [25]. During the last few decades, breeders in modern dairy cattle breeding programs have implemented strong artificial selection. Kim et al. showed that selection increases overall autozygosity across the genome, whereas the autozygosity in an unselected line does not change significantly across most chromosomes in cattle populations [26]. Genomic regions under positive selection show increased $\mathrm{ROH}$ levels due to local reductions in haplotype diversity, i.e. selective sweeps [27]. Certain haplotypes under these conditions constitute a large proportion of the total haplotype pool for the portions of the genome targeted for selective sweeps. In these conditions, a portion of the $\mathrm{ROH}$ is the actual selection target and is retained in the $\mathrm{ROH}$ region [28]. These $\mathrm{ROH}$ will increase in frequency and be subject to purging, as the spread of haplotypes carrying deleterious recessive alleles are restrained by forces counteracting the selection pressure driving the selective sweep [29]. Therefore, we expect that this $\mathrm{ROH}$ type would be on average shorter, composed of more common haplotypes, be concentrated in fewer genomic locations, and contain fewer deleterious alleles than the genomic average.

Short $\mathrm{ROH}$ are generally due to older haplotype relatedness, while longer $\mathrm{ROH}$ result from more recent parental relatedness [30]. Thus, short and medium sized $\mathrm{ROH}$ have been subject to selection for a longer period of time than longer $\mathrm{ROH}$. Furthermore, recombination will have had more time to trim down $\mathrm{ROH}$ that have been the target of selective sweeps [26, 31]. ROH regions are expected to exhibit an increased frequency of homozygotes compared to non- $\mathrm{ROH}$ regions, as the homozygote allelic frequency in non- $\mathrm{ROH}$ regions is $\mathrm{p}^{2}$ and the allelic frequency in $\mathrm{ROH}$ regions is $\mathrm{p}$, given a population allelic frequency of $\mathrm{p}$. Therefore, IBD results in enrichment of homozygotes in $\mathrm{ROH}$. Given the nature of cattle breeding, artificial selection is expected to play a more crucial role in shaping the frequency and distribution of functional variants in $\mathrm{ROH}$ in modern cattle relative to human populations.

Therefore, we expect that selection pressures could reduce the number of regions with an increased frequency of deleterious variations, and, at the same time, enrich for short $\mathrm{ROH}$ regions with substantial beneficial effects in cattle breeding populations. This remains an ongoing process, as the properties of genomic variants within $\mathrm{ROH}$ regions continue to be discovered in cattle [32-34]. An increase in functional variant frequency in different $\mathrm{ROH}$ length classes should also be examined under different population-genetic processes, such as the number of generations of inbreeding [30].

Genome scale bioinformatics annotations are available from a number of sources, including the Variants Effect Predictor from ENSEMBL [35], with several available levels of annotation. Generally, synonymous polymorphisms are the least subject to selective forces as they have the least effect on the resultant protein. Tools such as SIFT can be used to predict non-synonymous change 
effects on proteins, and, thus, give an idea of their likely selective pressure [36]. SIFT classifies non-synonymous changes as either non-deleterious or deleterious, based on the predicted effect on the protein. By comparing these classes (synonymous, non-deleterious non-synonymous, and deleterious non-synonymous), we can study how selection has shaped deleterious variants within the cattle genomic $\mathrm{ROH}$.

We hypothesize that due to strong artificial selection pressures and demographic processes in cattle, deleterious variants increased in frequency within $\mathrm{ROH}$ compared with non-deleterious variants and that the deleterious allelic frequency and distribution in $\mathrm{ROH}$ classes differs markedly from the human genome. Testing this hypothesis will contribute to our understanding of inbreeding in cattle and help elucidate how artificial selection and other population level processes affect the distribution of functional variants. To accomplish this, we examined patterns of $\mathrm{ROH}$ detected using full genome sequencing in four Danish cattle breeds and studied the distribution and frequency of deleterious and non-deleterious variations in different length $\mathrm{ROH}$ regions.

\section{Results}

\section{General statistics}

Runs of homozygosity (ROH) in the autosomes of 104 resequenced individuals were determined from four Danish dairy cattle breeds: Holstein (HOL), Jersey (JER), old Red Danish Dairy cattle (old-RED), and New Danish Red Dairy cattle (new-RED) (Fig. 1 and Additional file 1: Figure S1). The average genomic $\mathrm{ROH}$ content was $19.5 \%$ across the four cattle breeds, with HOL, JER, New-RED, and Old-RED having $18.67 \%$, $24.23 \%$, $11.84 \%$, and $23.26 \%$, respectively. The average number of $\mathrm{ROH}$ per genome was $715.5 \pm 21.0$, with an average size of $750,564.2 \mathrm{bp}$, ranging from $10 \mathrm{kbp}$ (the minimum size considered) to 49,290 kbp (Additional file 10: Table S1). The mean $\mathrm{ROH}$ size varied significantly between $\mathrm{HOL}$, JER, Old-RED, and New-RED $(P<0.001)$ with the exception of JER and Old-RED (Fig. 1c). The mean number of ROH was significantly different between HOL, JER, OldRED, and New-RED cattle $(P<0.001)$ with the exception of HOL and Old-RED (Fig. 1d).

The average genome-wide nucleotide diversity $(\pi)$ was 1.59 ( \pm 0.024$)$ heterozygous positions per kbp across all individuals, including $\mathrm{ROH}$, and 1.89 ( \pm 0.018 ) heterozygous positions per kbp across all individuals in the genome excluding $\mathrm{ROH}$ ( $\pi$-out) (Additional file 10: Table S1). The minimum and maximum nucleotide diversities were $1.50( \pm 0.029) \mathrm{SNPs} / \mathrm{kbp}$ in JER and $1.71( \pm 0.024) \mathrm{SNPs} /$ kbp in New-RED, respectively (Fig. 1a and Additional file 2: Figure S2, Additional file 10: Table S1) and were significantly different between all cattle breeds except HOL and New-RED $(P<0.05)$. $\pi$-out was significantly different between Old-RED and HOL, JER, and New-RED $(P<0.001$, two-tailed $t$-test) (Fig. 1a and b). Nucleotide

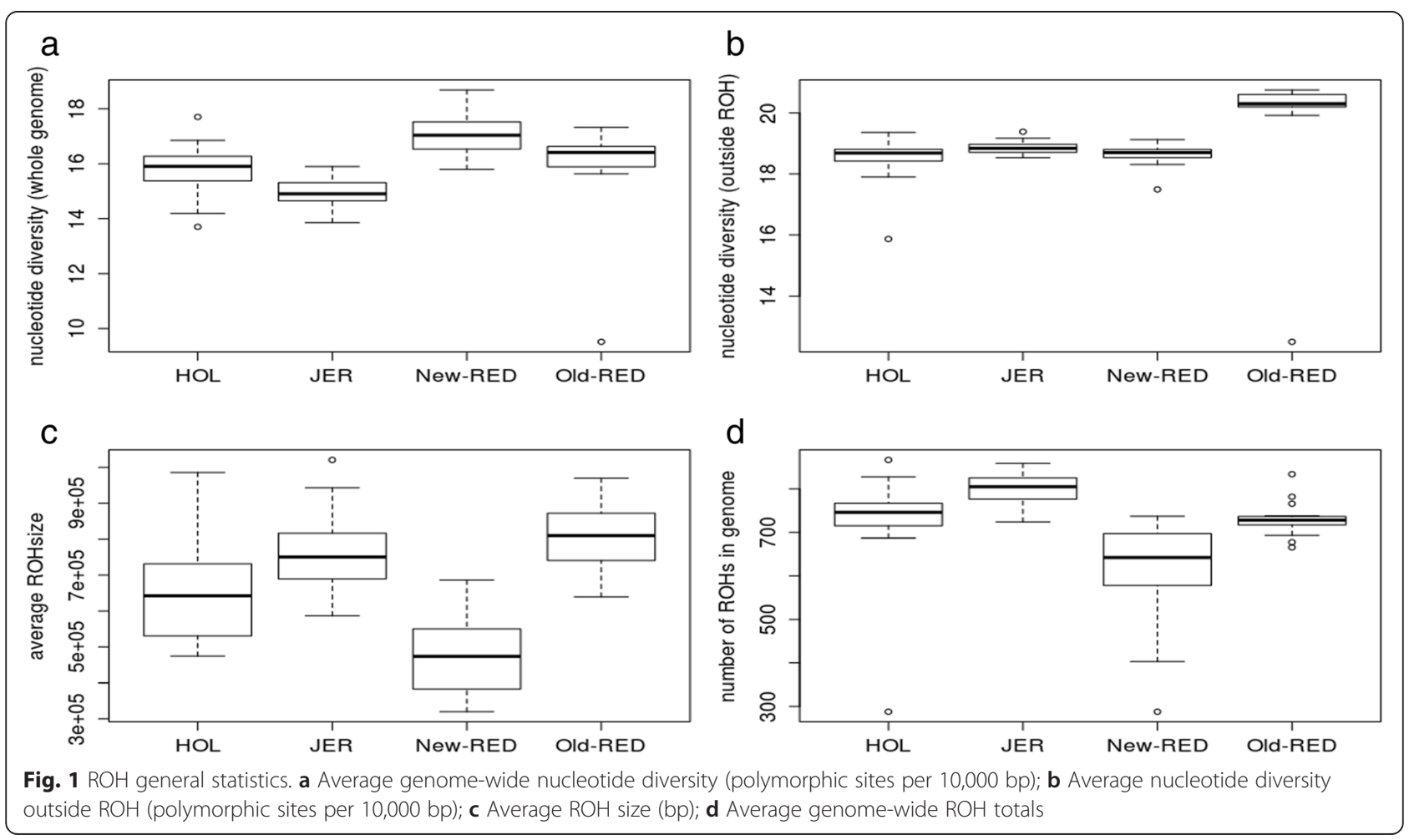


diversity was higher in the vicinity of the major histocompatibility complex (MHC) on chromosome 23 (Additional file 1: Figure S1).

\section{Genomic patterns of homozygosity}

$\mathrm{ROH}$ were separated into three size classes: small (10 kbp to $100 \mathrm{kbp}$ ), medium (0.1 to $3 \mathrm{Mbp}$ ), and large (> $3 \mathrm{Mbp}$ ) (as described in the Materials and Methods section). The proportion of $\mathrm{ROH}$ in each size class was computed in all 104 sequenced individuals. While small $\mathrm{ROH}$ were frequent throughout the genome, they constituted a small proportion of the entire genome (Fig. 2). In contrast, medium $\mathrm{ROH}$ were much less frequent, they constituted significantly more of the genome than either small or large $\mathrm{ROH}$. Large $\mathrm{ROH}$ were at least tenfold less numerous than medium $\mathrm{ROH}$, but nevertheless covered a sizable proportion of the total genome length. Old-RED cattle on average had the largest proportion of their genome in large $\mathrm{ROH}$. New-RED cattle had fewer genomic $\mathrm{ROH}$ and a smaller genomic proportion of $\mathrm{ROH}$ than HOL and JER cattle
$(P<0.001)$. Old-RED and JER cattle on average had more $\mathrm{ROH}$ and increased proportion of genomic homozygosity.

Principal component analysis successfully differentiated the four cattle breed individuals into different clusters based on SSR sequence data (Additional file 4: Figure S4), with JER the most distantly related based on PCA results. Three-dimensional plots did not show clear separation of cattle breeds based on to $\mathrm{ROH}$ size, $\mathrm{ROH}$ number, and $\pi$-out (Additional file 5: Figure S5). NewRED cattle represented the most variable cluster due to high nucleotide diversity and fewer ROH $(\mathrm{P}<0.001)$ (Fig. 1). There was lower nucleotide diversity and more total $\mathrm{ROH}$ in JER compared to HOL and New-RED (P < 0.001) (Fig. 1). Despite its most distant origin, JER had lower nucleotide diversity (Additional file 4: Figure S4 and Additional file 5: Figure S5), creating several clusters in the three dimensional plot (Additional file 5: Figure S5). However, all Danish cattle breeds were more or less clustered together, with the exception of two New-RED individuals (Additional file 5: Figure S5).

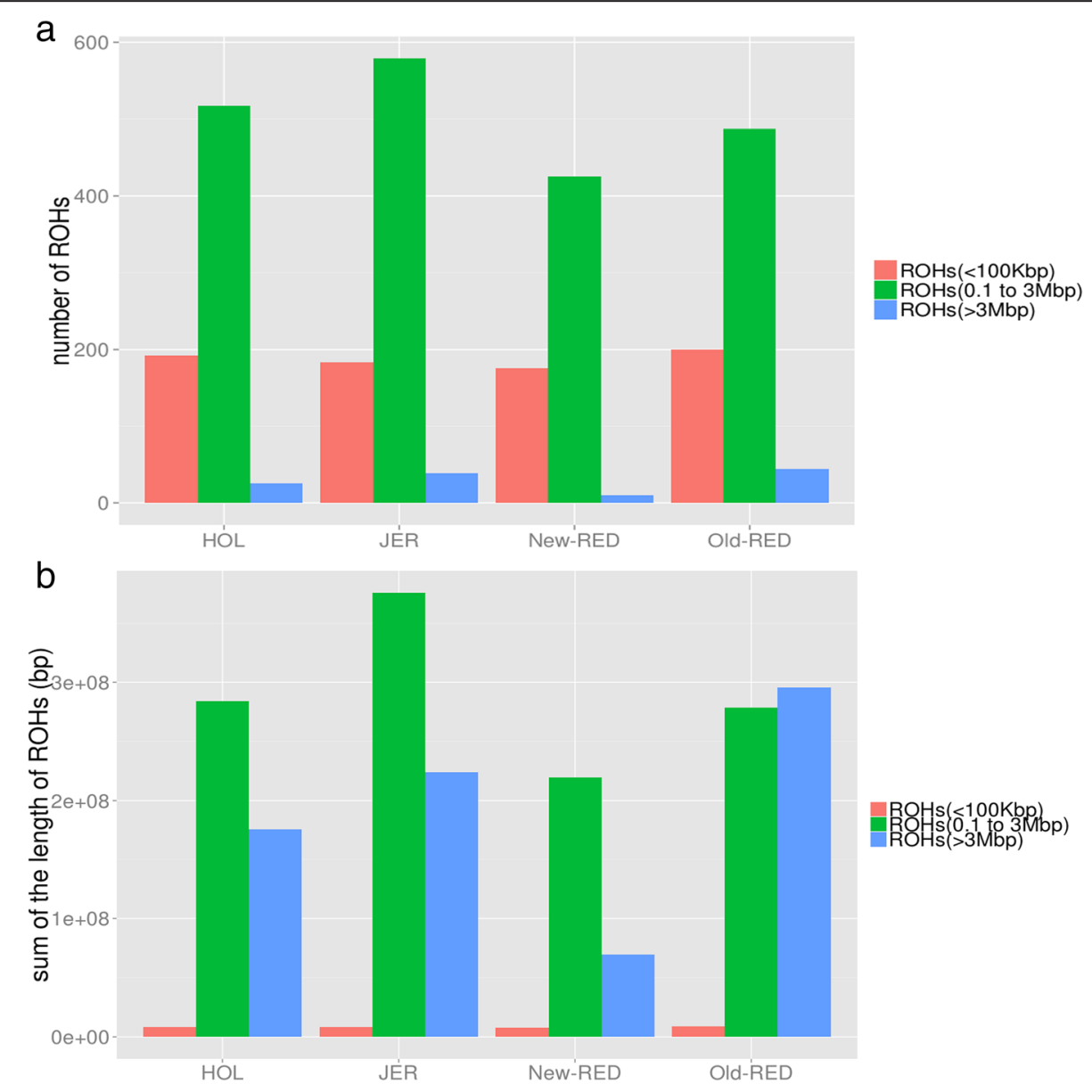

Fig 2 Total ROH number and genome proportions. a The average small (< $100 \mathrm{kbp}$, Red), medium ( 0.1 to 3 Mbp, Green), and large (> 3 Mbp, Blue) $\mathrm{ROH}$ numbers for the four breeds; $\mathbf{b}$ Average total genome $\mathrm{ROH}$ coverage for a given size class within each breed 
Based on sequence data, New-RED cattle exhibited the fewest $\mathrm{ROH}$ and smallest $\mathrm{ROH}$ sizes. This is a composite breed with contributions from other red breeds, including Swedish Red, Finnish Ayrshire, and Brown Swiss. Compared with New-RED, these data suggest that the Old-RED breed has been more inbred based on relatively high coverage of long regions of homozygosity (> $3 \mathrm{Mbp}$ ) (Additional file 5: Figure S5), probably due to a relatively small breeding population and recent years of close mating.

Furthermore, the sharing of $\mathrm{ROH}$ regions was examined among sequenced individuals (Additional file 15: Figure S10B and Additional file 16: Figure S11C). Sharing of $\mathrm{ROH}$ regions primarily happened in short rather than long $\mathrm{ROH}$ regions, likely a result of combination of inbreeding and selection. Significant correlations were observed between Fst, iHS, and shared ROH regions in bins of $500 \mathrm{~kb}$ compared with the whole genome average $(\mathrm{p}<0.001)$ (Additional file 15: Figure S10C and Additional file 16: Figure S11D). We also observed that instead of randomly distributed over the genomes (Additional file 17: Figure S12B), there were several obvious $\mathrm{ROH}$-dense peaks distributed shared between individuals across genomes (Additional file 17: Figure S12A). Therefore, the distribution of $\mathrm{ROH}$ is not only result of pure demography, but likely the result of selection.

\section{Distribution of functional variants in $\mathrm{ROH}$ regions} Number of deleterious homozygous genotypes in $\mathrm{ROH}$

Additional file 11: Table S2 and Additional file 12: Table S3 show the counts for reference homozygotes $(0 / 0)$, heterozygotes $(0 / 1)$, and non-reference homozygotes (1/1) at deleterious and non-deleterious sites, respectively, that were contained within $\mathrm{ROH}$ and non- $\mathrm{ROH}$ regions (all $g_{i, j}^{d, k}$ and $\left.g_{i, j}^{n, k}\right)$. The number of deleterious non-reference homozygotes was consistent with the $\mathrm{ROH}$ coverage in all four breeds. Old-RED and JER showed increased ROH coverage, with a higher number of non-reference deleterious homozygotes in the genome. Non-reference non-deleterious homozygotes also exhibited the same trends as deleterious homozygotes.

Figure 3 shows the total number of deleterious nonreference homozygotes $(1 / 1)$ as a function of the total proportion of the genome covered by $\mathrm{ROH}\left(G_{i, R}\right)$ for all sequenced individuals. As $\mathrm{ROH}$ coverage increased (high $G_{i, R}$ values), a greater number of homozygotes were observed within $\mathrm{ROH}$, which was consistent with findings

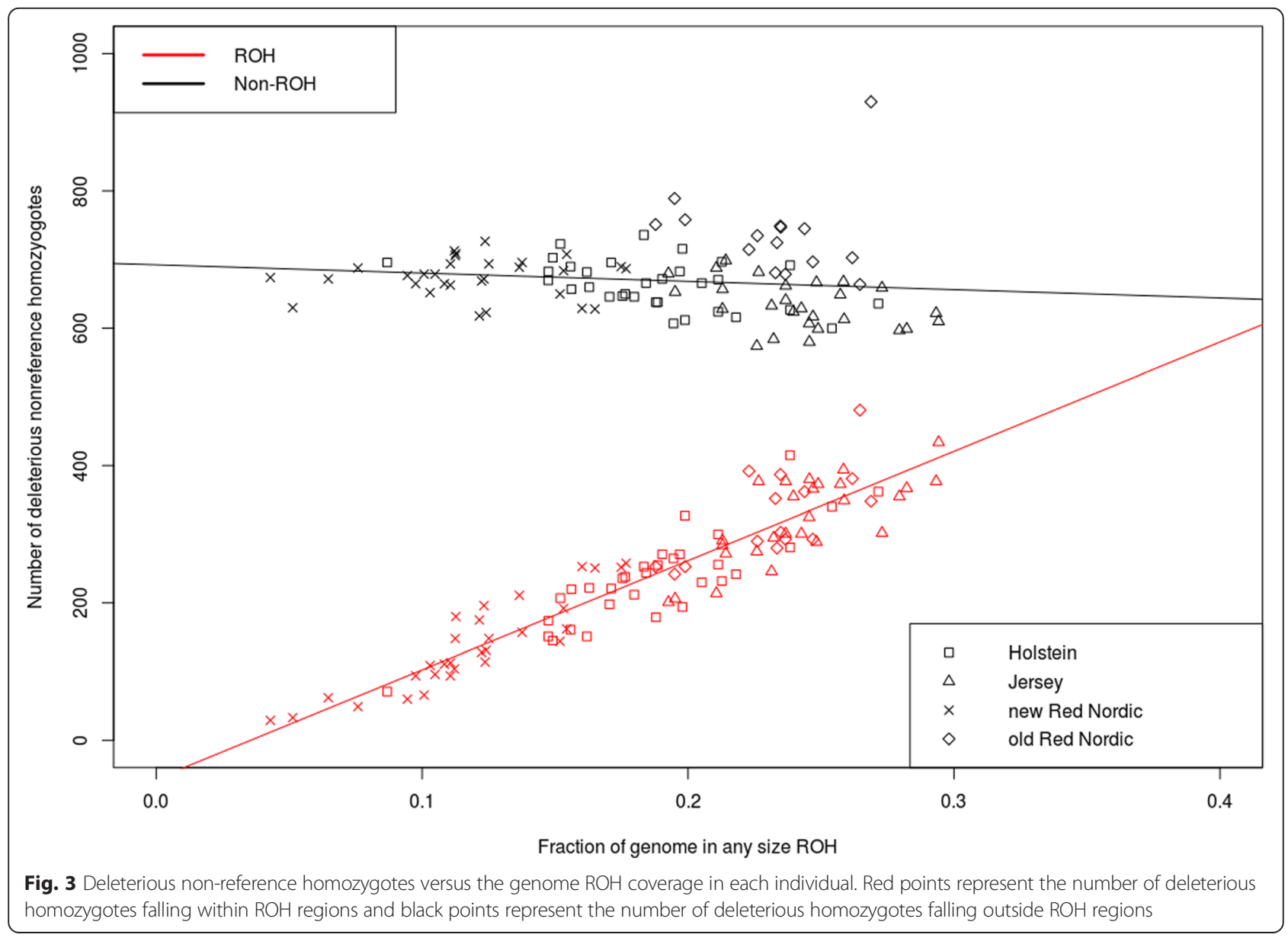


from the human genome [24]. There was a very strong positive correlation between the number of deleterious homozygotes and the genomic $\mathrm{ROH}$ proportion (Pearson $r=0.93$, slope $=1568.76$, intercept $=-57.63$ ). Similarly, the number of homozygotes outside of $\mathrm{ROH}$ decreased with the genomic $\mathrm{ROH}$ proportion due to smaller non- $\mathrm{ROH}$ regions as $\mathrm{ROH}$ coverage increased. As expected, there was a weak negative correlation between deleterious homozygotes outside $\mathrm{ROH}$ and the genomic $\mathrm{ROH}$ proportions (Pearson $r=-0.12$, slope $=-98.67$, intercept $=693.63$. . Compared with data from the human genome, the decreased slope for non- $\mathrm{ROH}$ regions was much shallower than the increased slope for $\mathrm{ROH}$ regions [24]. However, this indicates that the increased deleterious homozygotes in $\mathrm{ROH}$ regions exceed deleterious homozygote declines in non- $\mathrm{ROH}$ regions in cattle. Similar to the human genome [24], the fitted lines also predict that, on average, individual non-inbred cattle $\left(G_{i, R} \approx 0\right)$ carry approximately 694 deleterious homozygous variants. An increased in $\mathrm{ROH}$ coverage by $10 \%$ will increase the expected deleterious homozygote numbers in $\mathrm{ROH}$ regions by 157 and decrease the expected number of deleterious homozygotes in non- $\mathrm{ROH}$ regions by 10 , yielding an expected net increase of 147 deleterious homozygotes.

\section{Deleterious and non-deleterious homozygotes in $\mathrm{ROH}$ of any size}

Figure 4a shows the proportion of deleterious nonreference homozygotes inside and outside $\mathrm{ROH}$ regions $\left(f_{i, R}^{d}\right.$ and $\left.f_{i, R}^{n}\right)$ versus total genomic ROH coverage $\left(G_{i, R}\right)$. The proportions of non-deleterious and deleterious homozygous genotypes within $\mathrm{ROH}$ were strongly positively

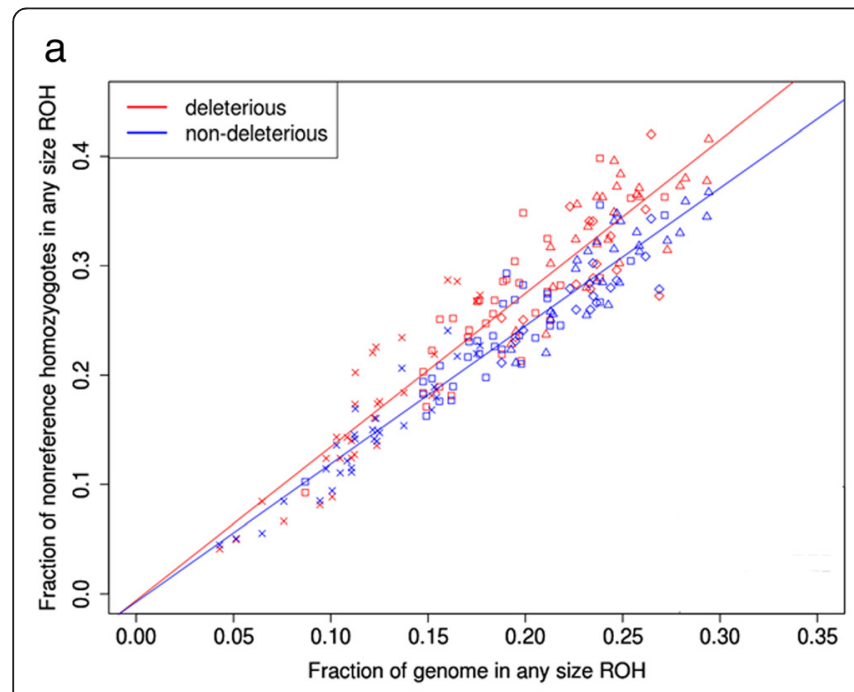

$\mathrm{b}$

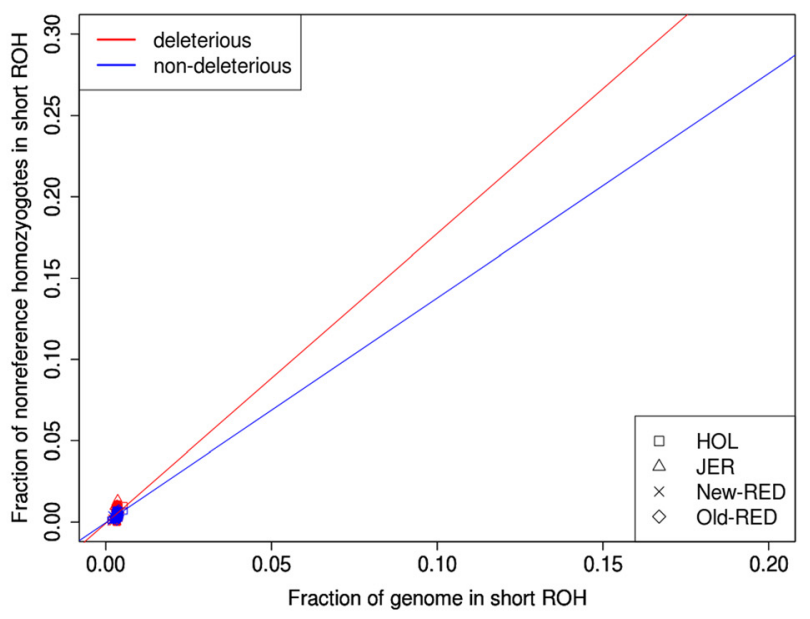

C

\section{d}
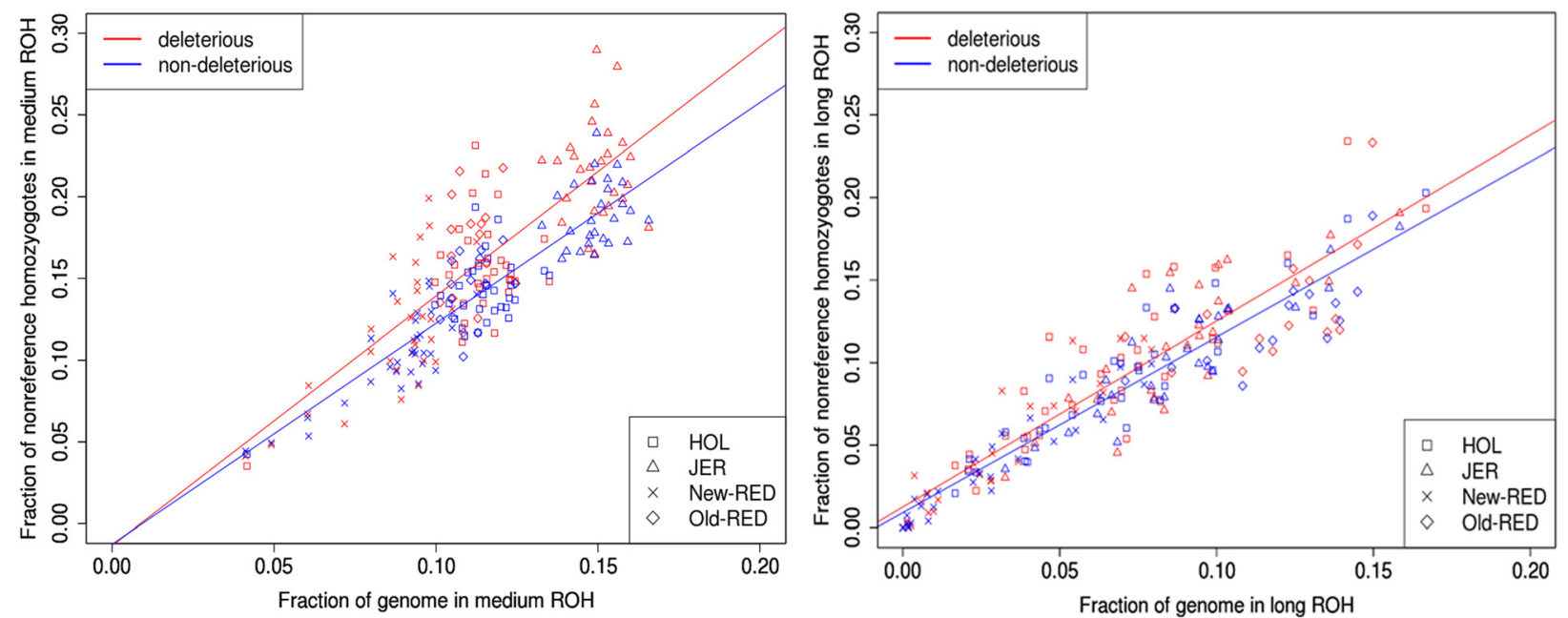

Fig. 4 The proportion of all genome-wide non-reference homozygotes falling in $\mathrm{ROH}$ regions versus the genome $\mathrm{ROH}$ coverage for each individual. a Any $\mathrm{ROH}$ region; b Short; c Medium; and $\mathbf{d}$ Long ROH regions. Red points represent deleterious homozygotes, and blue points represent non-deleterious homozygotes 
correlated with total genomic $\mathrm{ROH}$ coverage (Pearson $r=0.96$ for non-deleterious and $r=0.99$ for deleterious). These high correlations were expected, because as larger proportions of homozygous genotypes occur, $\mathrm{ROH}$ coverage in the genome increases, and therefore $\mathrm{ROH}$ comprise an increasingly greater proportion of the genome [24]. The $f_{i, R}^{d}$ proportion in genome-wide deleterious homozygotes within $\mathrm{ROH}$ consistently exceeded the $f_{i, R}^{n}$ proportion of genome-wide non-deleterious homozygotes within $\mathrm{ROH}$ and the increasing slopes differed between deleterious and non-deleterious variants.

After fitting the two linear regression models, we found $\beta_{3}$ was significant $(P<0.05)$ indicating that the interaction between the two regression slopes (deleterious and non-deleterious) was significant. However, $\beta_{2}$ was not significant $(P=0.9174)$ between the two regression intercepts. This is consistent with previous findings in humans [24], where deleterious homozygotes showed increased frequency in $\mathrm{ROH}$ relative to non-deleterious homozygotes and regression slopes were significantly different between deleterious and non-deleterious homozygotes.

\section{Deleterious and non-deleterious homozygotes by $\mathrm{ROH}$ size} Figure $4 \mathrm{~b}$ shows $f_{i, S}^{d}$ and $f_{i, S}^{n}$ versus total genomic coverage for small $\mathrm{ROH}\left(G_{i, S}\right)$ (Additional file 6: Figure S6). The proportion of non-deleterious homozygous genotypes in small $\mathrm{ROH}$, and the proportion of deleterious homozygous genotypes in small $\mathrm{ROH}$ were positively correlated with genomic coverage (non-deleterious Pearson $\mathrm{r}=0.32$, deleterious Pearson $\mathrm{r}=0.44)$. Figure $4 \mathrm{c}$ and $\mathrm{d}$ showed $f_{i, M}^{d}$ and $f_{i, M}^{n}$ versus total genomic coverage for medium $\left(G_{i, M}\right)$ and large $\left(G_{i, L}\right) \mathrm{ROH}$. The regressions for homozygote numbers in medium (non-deleterious $\mathrm{r}=0.88$, and deleterious $\mathrm{r}=0.80$ ) and large (non-deleterious $r=0.94$ and deleterious $r=0.90$ ) $\mathrm{ROH}$ had smaller $P$-values than in small $\mathrm{ROH}$.

These results show that deleterious homozygotes occur more frequently in $\mathrm{ROH}$ than non-deleterious homozygotes. Additionally, when the proportion of deleterious homozygotes within large $\mathrm{ROH}\left(f_{i, L}^{d}\right)$ is compared to the proportion within small $\mathrm{ROH}\left(f_{i, S}^{d}\right)$, there was a substantially higher proportion of genome-wide deleterious homozygotes in small and medium vs. large $\mathrm{ROH}$ especially in individuals with moderate to high $\mathrm{ROH}$ coverage proportions (Fig. 5). Given that ROH coverage (Fig. 1) for all individuals across the four breeds differed (as previously mentioned). Therefore, statistical tests for each size group were robust across the breeds and there were different $\mathrm{ROH}$ coverage groups across all individuals (Additional file 10: Table S1). Similar trends were observed for each $\mathrm{ROH}$ size group, and significantly different degrees of enrichment were observed within each size group.

The intercepts and slopes of deleterious homozygotes and non-deleterious homozygotes were significantly different for large and medium $\mathrm{ROH}\left(\beta_{2}=0.02590, P<0.05\right.$; $\left.\beta_{3}=-0.39931, P<0.001\right)$, but slopes and intercepts were not significantly different between small and medium $\mathrm{ROH}$ $\left(\beta_{2}=-0.0126778, P=0.433 ; \quad \beta_{3}=-0.2562209, \quad P=0.948\right)$. These results indicate inbreeding that generates short and medium $\mathrm{ROH}$ increases the proportions of deleterious and non-deleterious homozygotes in $\mathrm{ROH}$ regions compared to long $\mathrm{ROH}$.

If deleterious, non-deleterious, and synonymous homozygotes are considered as three separate classes, patterns similar to those observed in the deleterious and

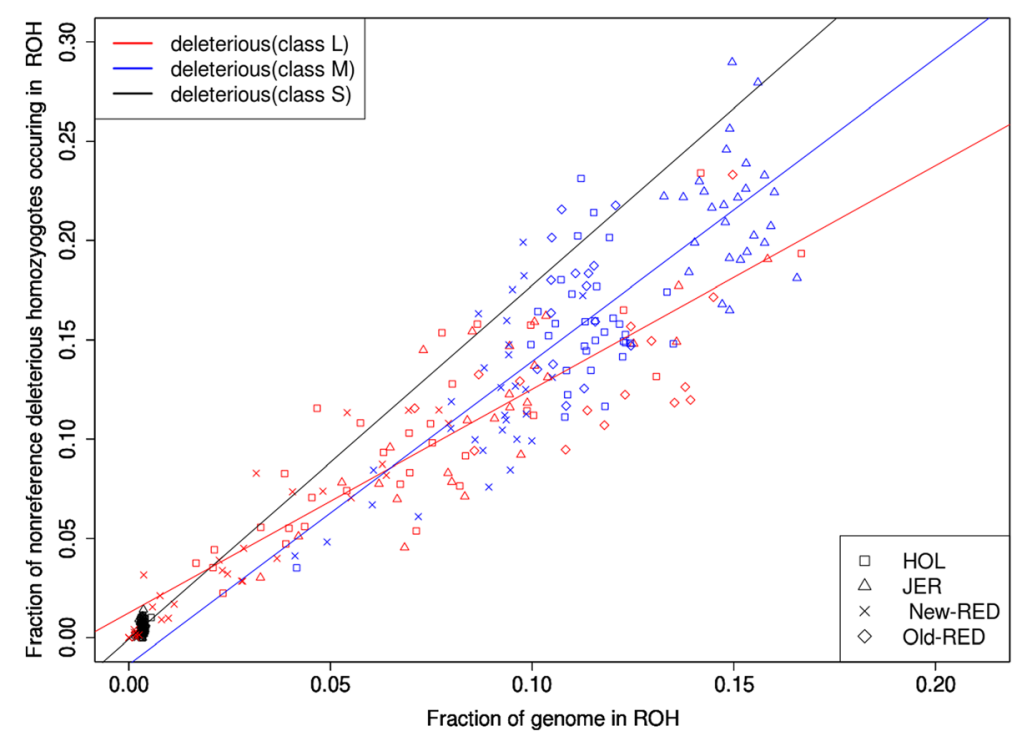

Fig. 5 The genome-wide proportion of all non-reference homozygotes falling in different $\mathrm{ROH}$ sizes versus genome ROH coverage for each individual. Red, orange, and black points represent deleterious homozygotes in large, medium, and small ROH regions, respectively 
non-deleterious homozygotes analysis emerge. Deleterious homozygotes were at highest proportions in short and medium length $\mathrm{ROH}$. There were smaller proportions of synonymous and non-deleterious homozygotes as genomic $\mathrm{ROH}$ coverage of small and medium $\mathrm{ROH}$ increases (Additional file 7: Figure S7 and Additional file 8: Figure S8). In large $\mathrm{ROH}$, the synonymous homozygote proportion over all homozygotes was higher than deleterious and non-deleterious homozygote proportions.

\section{Nonsense variants and $\mathrm{ROH}$}

Additional file 13: Table S4 and Additional file 14: Table S5 report nonsense and loss of function nonsense sites, respectively, with counts for reference homozygotes $(0 / 0)$, heterozygotes $(0 / 1)$, and non-reference homozygotes $(1 / 1)$, which fell into $\mathrm{ROH}$ and non- $\mathrm{ROH}$ regions. Figure $6 \mathrm{a}$ shows nonsense mutation distribution across all $\mathrm{ROH}$. For low- $\mathrm{ROH}$ individuals, the mean proportion of nondeleterious homozygote variants falling in $\mathrm{ROH}$ marginally exceeded the nonsense or loss of function variants. For high- $\mathrm{ROH}$ individuals, however, the proportion of non-deleterious homozygotes within $\mathrm{ROH}$ was lower than for nonsense homozygotes. When $\mathrm{ROH}$ are segregated by size (Fig. 6b-d), including individuals with high genomic $\mathrm{ROH}$ coverage, the proportion of nonsense homozygotes in medium $\mathrm{ROH}$ was greater than that of non-deleterious homozygotes, while the proportion of nonsense homozygotes was slightly lower than nondeleterious homozygotes for large $\mathrm{ROH}$. This is consistent with the finding that high- $\mathrm{ROH}$ individuals exhibited an increased proportion of damaging homozygotes (nonsense mutations) in $\mathrm{ROH}$ of any size (Fig. 6a), primarily driven by medium ROH (Fig. 6d).

\section{Discussion}

\section{Cattle genomic ROH patterns}

The 104 individuals re-sequenced in the present study are key ancestors from the four Danish cattle breeds. The New-RED is a composite breed with its primary origins in the Old Danish Red, and includes contributions from other red breeds, including Swedish Red Finnish Ayrshire, and Brown Swiss cattle [37]. Our results showed $\mathrm{ROH}$ size ranged from tens of $\mathrm{kb}$ to several $\mathrm{Mb}$ and varied among individuals from different cattle breeds. Overall, medium sized $\mathrm{ROH}$ were most common. The average proportion of the genome represented by $\mathrm{ROH}$ was $19.5 \%$ across all sequenced individuals. However, pedigree records and lower density SNP chips underestimated the

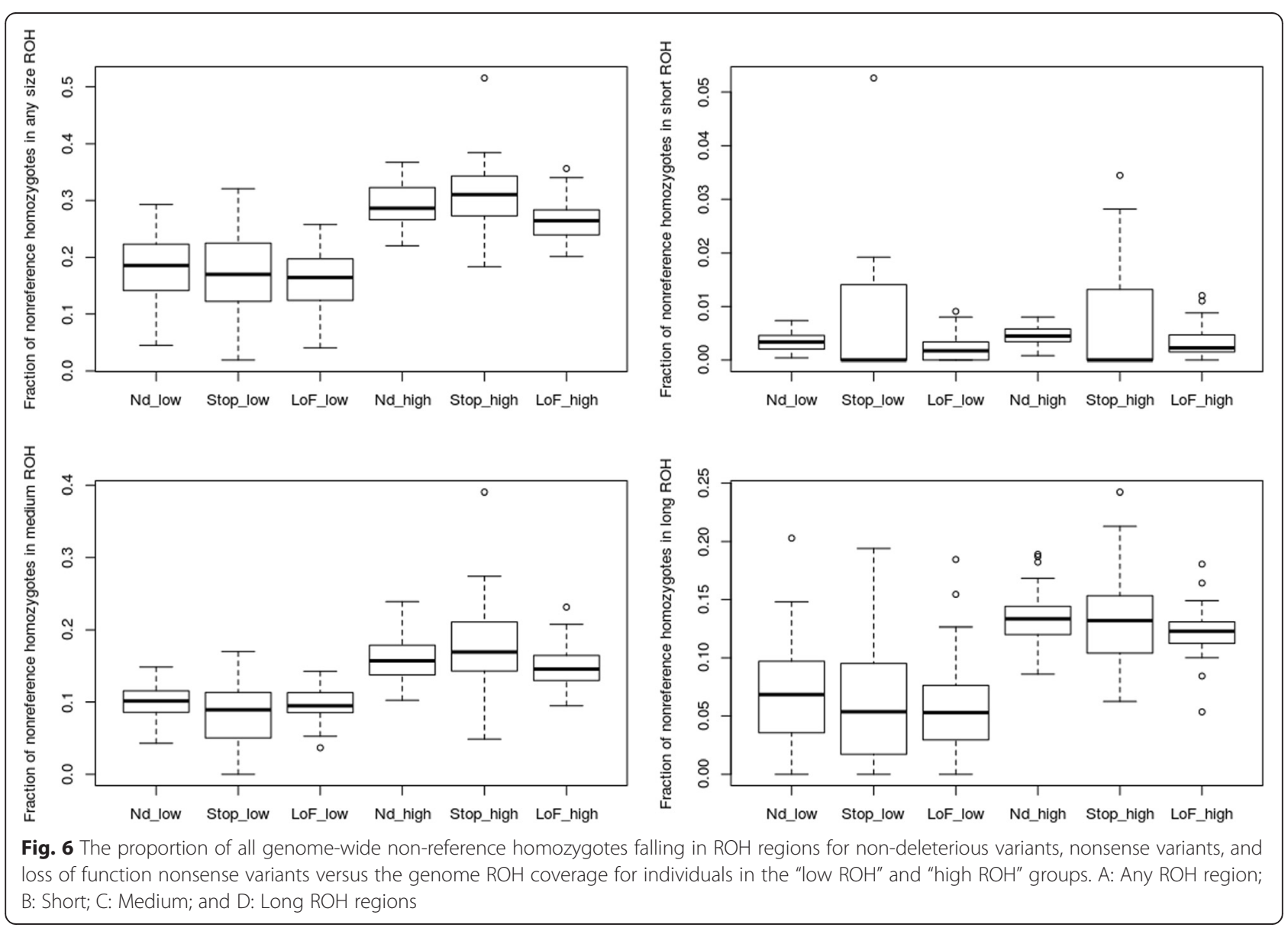


inbreeding coefficient for these 104 bulls compared with that generated using $\mathrm{ROH}$ from genome sequencing. A previous study in Danish cattle also reported a less than $5 \%$ inbreeding coefficient using pedigree information [6]. Among breeds, JER and Old-RED were relatively more inbred compared with HOL based on high $\mathrm{ROH}$ genome coverage. Meanwhile, a high number and proportion of long $\mathrm{ROH}$ were detected in Old-RED, likely indicating a small population with close mating for some period of time. However, the smallest proportion of $\mathrm{ROH}$ was detected in New-RED, presumably due to its outbred origins. These $\mathrm{ROH}$ patterns are consistent with the known population history for these four breeds [37]. Pemberton et al. allocated humans from different demographics to their corresponding place of origin by inferring population history from $\mathrm{ROH}$ analysis [28]. In our study, we also determined that individuals with similar $\mathrm{ROH}$ distributions clustered together due to similar patterns of variation (Additional file 5: Figure S5). In contrast, PCA generated different cluster types in the same sets of individuals based on population structure (Additional file 4: Figure S4).

The fact that nucleotide diversity outside $\mathrm{ROH}$ was higher in Old-RED than HOL, JER, and New-RED indicates that the historic genetic diversity of Old-RED might be relatively higher than the other breeds examined due to larger breeding populations for each of several past generations. Furthermore, evidence suggested Old-RED was the source population of New-RED. Although the newly derived RED populations exhibited the highest nucleotide diversity across the entire genome, the ancient haplotypes were more diverse in the ancestral Old-RED population. Reduced nucleotide diversity outside $\mathrm{ROH}$ in New-RED may also be explained by gene flow from HOL to New-RED. In addition, nucleotide diversity levels outside $\mathrm{ROH}$ presumably reflected the different origin of Old-RED from HOL and JER.

Bovine major histocompatibility complex (MHC) regions have long been known in the cattle genome [38, 39]. Our analysis also detected MHC regions, which have a high degree of nucleotide diversity, on chromosome 23 (Additional file 1: Figure S1). Pemberton et al. reported a correlation between linkage disequilibrium levels and $\mathrm{ROH}$ distribution in the human genome [28]. MHC regions contain recombination hotspots $[38,40]$ and, therefore, high recombination rates to maintain relatively high levels of genetic diversity, but are subject to over dominant selection [38]. $\mathrm{ROH}$ are rarely present in $\mathrm{MHC}$ regions of the cattle genome, preventing random distribution of $\mathrm{ROH}$ (Additional file 1: Figure S1).

Purfield et al. [19] showed ROH patterns in cattle populations using SNP chip data. Our analysis is the first to use next-generation sequencing data to infer $\mathrm{ROH}$ in cattle and indicate that long $\mathrm{ROH}$ can only be detected with $50 \mathrm{k}$ or HD SNP chip data (minimum $\mathrm{ROH}$ size was $1 \mathrm{Mb}$ ), while short $\mathrm{ROH}$ are not detectable due to the required density of SNP chip data (Additional file 3: Figure S3). Short ROH regions shared between individuals in our data (Additional file 15: Figure S10b and Additional file 16: Fgure S11c) confirm that short $\mathrm{ROH}$ were selected and derived from ancient haplotypes that became fixed in populations (Additional file 9: Figure S9), while long $\mathrm{ROH}$ are the result of more recent inbreeding events. Consequently, SNP chip data misses information more relevant to historic inbreeding practices rather than recent inbreeding events. The significant correlation between the sharing of $\mathrm{ROH}$ regions and regions putatively under selection (from Fst analysis and iHS testing) (Additional file 15: Figure S10C and Additional file 16: Figure S11D) suggests that some of these short shared $\mathrm{ROH}$ are the result of a combination of inbreeding and selection. Instead of $\mathrm{ROH}$ regions randomly distributed over the genome, there were dense- $\mathrm{ROH}$ peak regions in the actual sharing of $\mathrm{ROH}$ regions among individuals (Additional file 17: Figure S12), which supports the hypothesis that the observed $\mathrm{ROH}$ patterns are not solely a result of demography (Additional file 17: Figure S12), as random $\mathrm{ROH}$ distributions would only be expected as a result of inbreeding.

\section{Distribution of functional variants in the cattle genome}

Years of intensive artificial selection in cattle breeding have resulted in reduced genetic diversity in cattle populations as demonstrated by the high proportion of $\mathrm{ROH}$ $(11.8$ - $24.2 \%$, average $19.5 \%)$ found in this study. However, the results regarding $\mathrm{ROH}$ patterns suggest that the distribution and enrichment of putative functional variants in different $\mathrm{ROH}$ lengths is more interesting. Szpiech et al. suggested that damaging variants are more enriched in human $\mathrm{ROH}$, particularly longer $\mathrm{ROH}$ [24]. The observed speed of deleterious homozygote accumulation in $\mathrm{ROH}$ far exceeds the accumulated decrease in deleterious homozygotes in non- $\mathrm{ROH}$ regions (Fig. 3). This is expected, since identity by decent causes homozygosity to increase in $\mathrm{ROH}$ regions compared to non- $\mathrm{ROH}$ regions. Deleterious variants were expected at a low frequency; therefore, rare occurrences of deleterious alleles were expected in the homozygous state. However, when a stretch of homologous DNA fragments are identical by descent, the probability of deleterious alleles increases (at a rate of $\mathrm{p}$ rather than $\mathrm{p}^{2}$, where $\mathrm{p}$ is allelic frequency). We also observed a higher allele frequency of deleterious variants in $\mathrm{ROH}$ regions compared to non-deleterious variants (Fig. 4). This was also expected as increased deleterious variants occur when allele frequencies are extreme, as has been observed in humans [24]. In cattle, variants with 'deleterious' effects on protein structure may be artificially selected more frequently due to economic benefits. One example of this is a myostatin 
gene mutation in Belgian Blue cattle resulting in a "double muscling" phenotype. Meat from these cattle has a reduced fat content, as the mutation converts feed into increased lean muscle [41].

Cattle populations have been under strong artificial selection for many generations. The significant correlation between sharing of $\mathrm{ROH}$ regions and regions putatively under selection pressure (from Fst analysis and iHS testing) (Additional file 15: Figure S10 and Additional file 16: Figure S11) confirms that some of the shared short $\mathrm{ROH}$ regions have been selected and spread throughout the population. Moreover, by randomization of $\mathrm{ROH}$ regions over the genomes, Additional file 17: Figure S12B presented the patterns of $\mathrm{ROH}$ were only result of inbreeding. However, we do observe several dense-ROH peak regions shared among individuals in our populations, further supporting our belief that $\mathrm{ROH}$ patterns are not only the result of pure demography. Therefore, the distribution pattern and abundance of functional variants in different $\mathrm{ROH}$ lengths in cattle likely differs from the human population. Artificial selection purges deleterious alleles from regions that frequently occur in $\mathrm{ROH}$, favoring alleles with strong beneficial effects. Specifically, the interaction between inbreeding and artificial selection for particular variants can have a strong effect on the distribution of functional variants. Moreover, potentially deleterious mutations might hitchhike with selected variants. Long-term artificial selection enriches cattle populations with beneficial alleles in short and medium $\mathrm{ROH}$, along with hitchhiked deleterious variants, while variants in long $\mathrm{ROH}$ remain neutral.

The proportion of predicted deleterious homozygotes was greater in $\mathrm{ROH}$ regions than non-deleterious homozygotes. However, predicted deleterious homozygotes varied by the length of $\mathrm{ROH}$. The rate of change differed between small, medium, and long $\mathrm{ROH}$. Higher rates were observed in short and medium compared to long $\mathrm{ROH}$. The slopes for deleterious and non-deleterious homozygotes were significantly different in short and medium $\mathrm{ROH}$, and the patterns were similar for predicted nonsense (gain or loss of a stop codon) and loss of function (in-frame and frameshift) variants (Fig. 6). We also examined patterns of deleterious homozygote frequency in $\mathrm{ROH}$ using different length thresholds and saw the similar trends as reported using our original length thresholds (1.small $\mathrm{ROH}$ : length $<50 \mathrm{kbp}$; medium $\mathrm{ROH}$ : $50 \mathrm{kbp}<=$ length $<=2 \mathrm{Mbp}$; long $\mathrm{ROH}$ : length $>2$ Mbp 2. small ROH: length $<150$ kbp; medium $\mathrm{ROH}$ : 150 kbp < =length < =4 Mbp; long ROH: length > $4 \mathrm{Mbp}$ ).

Predicted deleterious variants may be detrimental, however, these allele frequencies cannot increase in long $\mathrm{ROH}$, as inbred individuals harboring a large proportion of long $\mathrm{ROH}$ with a high frequency of deleterious alleles will have reduced fitness, leading to decreased survival.
Alternatively, predicted deleterious variants may be harmful alleles that were carried into the genome with artificially selected beneficial alleles, and were therefore favored by selection over a number of generations and are reflected in short or medium $\mathrm{ROH}$ (Additional file 9: Figure S9, Additional file 15: Figure S10 and Additional file 16: Figure S11). Long $\mathrm{ROH}$ are evidence of recent shared ancestry, while short $\mathrm{ROH}$ typically reflect more ancient relatedness [30]. Long $\mathrm{ROH}$ regions have gone through selection for few generations, and will eventually break down into medium and then short $\mathrm{ROH}$. Allelic combinations will likely be recombined within a few generations and disappear due to segregation [15]. In contrast, deleterious short or medium $\mathrm{ROH}$ variants, which reportedly hitchhike with beneficial alleles, are thought to persist for an extended periods of time and are shared among individuals via gene flow (Additional file 9: Figure S9, Additional file 15: Figure S10 and Additional file 16: Figure S11) [15]. Some of these shared short $\mathrm{ROH}$ regions were observed to be overlapping with regions under selection based on the Fst analysis and iHS testing (Additional file 15: Figure S10 and Additional file 16: Figure S11). One mechanism for this is when a beneficial mutation occurs in a population and then spreads to the entire population, forming a selective sweep. Artificial selection will favor short or medium $\mathrm{ROH}$ regions harboring beneficial mutations that will spread and eventually become fixed in the sampled populations (Additional file 9: Figure S9, Additional file 15: Figure S10 and Additional file 16: Figure S11). Therefore, we deduce that some predicted deleterious homozygotes in short or medium $\mathrm{ROH}$ were deleterious alleles that hitchhiked with beneficial variants and were selected in the population. Alternatively, shorter $\mathrm{ROH}$ may represent the interplay between random inbreeding and artificial selection for particular variants. Therefore, some of these shared short $\mathrm{ROH}$ tended to be candidate regions for selection. However, homozygosity for certain short and medium $\mathrm{ROH}$ regions were not maintained due to the absence of selection for any specific alleles; therefore, variants with deleterious effects will be purged by artificial selection. It should also be noted that the confounding effect of inbreeding with selection in generating long stretch of haplotype homozygosity may influence the robustness of EHH-based tests.

Lohmueller et al. [29] suggested human populations with decreased genetic diversity supported an excess of recessive deleterious variants, resulting from founder effects in ancient populations during speciation [4], with inflation in the frequency of these rare variants in contemporary populations. We observed a higher proportion of deleterious than non-deleterious homozygotes in $\mathrm{ROH}$ (Fig. 4). Therefore, another possible explanation for these results is a history of population inbreeding 
and founder events (Additional file 9: Figure S9), with preservation of deleterious variants from ancient populations in contemporary cattle populations represented by our samples. However, artificial selection has been implemented in cattle populations for many years, and regions under selection pressure tend to overlap with short, shared $\mathrm{ROH}$ regions (Additional file 15: Figure S10 and Additional file 16: Figure S11). This suggests that these preserved ancient alleles may have carried deleterious alleles via hitchhiking.

Predicting how variation affects gene function has varying degrees of reliability. SIFT scores [36] were used to estimate potential fitness consequences for nonreference alleles in our analysis. Certainty regarding predicted functional effects is based on changes in the primary amino acids and impacts on protein function and biological processes. However, here we examined functional variant distribution in $\mathrm{ROH}$ regions instead of exploring the effects of each deleterious variant on fitness. A general pattern was obtained by combining all deleterious or non-deleterious variants into one specific class to explore variant distribution in $\mathrm{ROH}$ regions. Furthermore, our observations were confirmed by nonsense and loss of function variant classification. Similar patterns were observed when grouping variants into nonsense and loss of function variants. It should be noted we only emphasized non-reference homozygotes with substantial effects on the organism and did not determine the impacts of reference homozygotes. There is the potential for deleterious or selected reference homozygotes, and, therefore, these alleles should also be examined. However, reference alleles are not annotated with a SIFT score, preventing their examination in this study.

\section{Conclusion}

We characterized $\mathrm{ROH}$ using genome sequence data in four cattle breeds. The genome-wide proportion and distribution patterns of $\mathrm{ROH}$ differed among HOL, JER, New-RED, and Old-RED cattle breeds. We observed a significant correlation between the shared short $\mathrm{ROH}$ regions and regions putatively under selection. We also showed the distribution of functional variants in different $\mathrm{ROH}$ regions and an increased frequency in predicted deleterious homozygotes in short and medium, but not long, $\mathrm{ROH}$, which differs from the human genome. However, the observed pattern and distribution of functional variants is consistent with the population history of the cattle studied, and we suspect that the observed distribution of functional variants is a result of combination of inbreeding and long-term artificial selection in cattle populations. This is supported by the significant correlation between shared short $\mathrm{ROH}$ regions and regions putatively under selection. Our findings contribute to the understanding of the effects of inbreeding and probably selection on shaping the distribution of functional variants in the cattle genome.

\section{Methods}

As previously obtained cattle genomic sequences were used exclusively in this project and no live animal experiments were performed, no animal use and care committee approval was required.

\section{SNP genotyping, sequencing, variant calling, and quality control}

A total of 104 bulls, (i.e. 32 HOL, 27 JER, 15 old-RED, and 30 new-RED) with high genetic contributions to the current Danish dairy cattle populations, including Holstein (HOL), Jersey (JER), old Red Danish Dairy cattle (old-RED), and New Danish Red Dairy cattle (new-RED) were selected for sequencing. In addition, 81 and 85 individuals among those sequenced were respectively genotyped using High Density SNP assays (Infinium BovineHD BeadChip), and the $50 \mathrm{k}$ assay Infinium BovineSNP50 v.1 BeadChip (Illumina, San Diego CA). SNP genotyping was performed as described by Höglund et al. [42].

All selected individuals' genomes were sequenced to $\sim 10 \times$ depth using Illumina paired-end sequencing. Sequencing was undertaken at the Beijing Genomics (Shenzhen, China), Aros Applied Biotechnology A/S (Aarhus, Denmark), and at Aarhus University (Foulum, Denmark). Reads were aligned to the cattle genome assembly UMD3.1 [43] using bwa [44]. Aligned sequences were converted to raw BAM files using samtools [45]. Duplicate reads were removed using the samtools rmdup option [45]. The Genome Analysis Toolkit [46] was used for local realignment around insertion/deletion (indels) regions, and recalibration following the Human 1000 Genome guidelines incorporating information from dbSNP [47]. Finally, variants were called using the Genome Analysis Toolkit [46], which simultaneously calls short indels and SNPs by incorporating information from $d b S N P$ [48]. Indels were excluded in further analyses, and variants with phred scores exceeding 100 were included in nucleotide diversity calculations and $\mathrm{ROH}$ computations. Nucleotide diversity was calculated for bins of $10 \mathrm{kbp}$ over the entire genome in all $104 \mathrm{se}-$ quenced individuals following the procedures of Bosse et al. and Nei and Li $[15,49]$. SNP counts per $10 \mathrm{kbp}$ bin were corrected for the number of bases within a 10 kbp bin, which is proportional to 10,000 covered bases. A correction factor must be applied, significant portions $(0.5-2 \mathrm{x})$ were not covered. The correction factor equaled DP/bin size, where DP is the coverage in bp/bin.

\section{Principal component analysis}

Genotypes were extracted from the sequence data sets (32 HOL, 27 JER, 15 old-RED, and 30 new-RED bulls) 
following variant calling using a perl script. Bi-allelic variant calls with phred scores exceeding 100 and higher than average read depths were used in genotype construction. Genome-wide Complex Trait Analysis (GCTA) [50] was employed to construct a genetic relationship matrix for chromosome 1 using all sequenced individuals. In addition, the population structure was examined for four breeds using a principal component method [51] using GCTA [50].

\section{Runs of homozygosity}

The method developed by Bosse et al. [15] was applied to identify $\mathrm{ROH}$ on all autosomes of the 104 sequenced individuals. The threshold to declare a $\mathrm{ROH}$ was set to a SNP count maximum of $0.25 x$ the genome coverage following Bosse et al. [15]. ROH were also detected in $50 \mathrm{k}$ and HD chip genotyped animals using the Runs of Homozygosity tool in PLINK (v. 1.07) [52], with parameters similarly adapted to sequence data. Extracted $\mathrm{ROH}$ based on the technique of Bosse et al. [15] were compared with $\mathrm{ROH}$ calculated from PLINK (v. 1.07) [52] for the same individual and chromosome. $\mathrm{ROH}$ extracted from sequence data were further classified into three size categories: short $\mathrm{ROH}$ are smaller than $100 \mathrm{kbp}$, and reflect ancient homozygosity haplotypes; medium $\mathrm{ROH}$ exhibit sizes from 0.1 to $3 \mathrm{Mbp}$ and arise from relatedness within populations; and long $\mathrm{ROH}$ result from recently related individuals, with $\mathrm{ROH}$ sized larger than $3 \mathrm{Mbp}[15,30]$.

The distribution of functional variants in the cattle genome was detected by computing the proportion of an individual's genome covered by any sized $\mathrm{ROH}$ region ( $j=\mathrm{ROH}$ genome coverage; subscripts denote the $\mathrm{ROH}$ size). All comparison tests among breeds were twotailed $t$-tests performed with the t.test function in $\mathrm{R}$ (v.3.1.0). Correlation coefficient significance tests were evaluated using the cor and cor.test function in $\mathrm{R}$ (v.3.1.0). The sharing of $\mathrm{ROH}$ between individuals among HOL, JER, Old-RED and New-RED was computed by counting the overlap $\mathrm{ROH}$ regions between individuals in the $10 \mathrm{~kb}$ bin over the full length of genome. To examine if the $\mathrm{ROH}$ distribution is the result of pure demography, we randomized the number and length of $\mathrm{ROH}$ regions for each individual over the genome and re-distributed them randomly throughout their genomes. These were then compared with the actual sharing of $\mathrm{ROH}$ regions between individuals as previously described.

\section{Detection of selection signatures Fst analysis}

The genetic differentiation between individuals among HOL, JER, Old-RED and New-RED was measured by pairwise Fst analysis following Weir and Cockerham [53]. The pairwise Fst between the defined breeds was computed with Genepop 4.2 in bins of $10 \mathrm{~kb}$ over the full length of the genome [53]. Correlation between Fst and sharing of $\mathrm{ROH}$ averaged for the same bins of $500 \mathrm{~kb}$ was calculated with Pearson correlation in R (v.3.1.1).

\section{Extended haplotype homozygosity tests}

The extended haplotype homozygosity tests were implemented between the breeds for the sequenced individuals. The genome-wide scan for integrated haplotype score (iHS) within each breed was performed using the $\mathrm{R}$ package rehh $[54,55]$, and the four breeds were compared using the ies2rsb function in rehh [54, 56]. Finally, the significance levels (the corresponding $\mathrm{p}$ values, assuming $\mathrm{iHS}$ or $\mathrm{rSB}$ are normally distributed under the neutral hypothesis) between breeds were averaged for a bin of $500 \mathrm{~kb}$ and were correlated with $\mathrm{ROH}$ sharing for the same bin of $500 \mathrm{~kb}$ by Person's correlation.

\section{Variant annotations and classifications by predicted functional impacts}

The called variants of each genomic site were annotated using ENSEMBL (v.67) databases with Variant Effect Predictor (VEP) [35]. Any sites with multiple transcripts resulting in multiple annotations were annotated only once using the by-gene option in VEP [35]. VEP determines variant effects (i.e. SNPs, insertions, deletions, CNVs, or structural variants) on genes, transcripts, and protein sequence, as well as regulatory regions. It predicts genes and transcripts affected by variants, variant locations (e.g. upstream of a transcript, in a coding sequence, in non-coding RNA, in regulatory regions), and any variant consequence on protein sequence (e.g. gain or loss of a stop codon, missense, frameshift). SIFT scores were used to classify annotations for non-reference alleles. Given a set of mutations, SIFT predicts the potential effect a non-reference allele has on encoded proteins, and integrates effects of amino acid change, folded structure (predicted or known), and conservation score. SIFT categorizes the non-reference mutations as "deleterious" or "tolerated". In this analysis, we classified non-reference alleles with a "deleterious" predicted effect as "damaging", while "non-deleterious" or "tolerated" non-reference alleles were classified as "non-damaging". It should be noted that nonreference alleles predicted as "deleterious" could just be different from reference alleles (in cattle, the reference genome was constructed from a beef breed rather than a dairy breed), which could exhibit substantial effects on amino acid change. Non-reference homozygotes were compared between non-deleterious (non-damaging), deleterious (damaging), and synonymous groups. Although truly damaging alleles could falsely be classified as nondamaging, the objective of this analysis was to detect the distribution of functional variants in regions of homozygosity at the whole genome level. 
Distribution of functional variants in $\mathrm{ROH}$ regions Number of deleterious homozygous genotypes in $\mathrm{ROH}$

We followed the method proposed by Szpiech et al. [24] to detect predicted functional variant distribution in $\mathrm{ROH}$ regions in these three cattle breeds [57]. We partitioned genotypes in our data into those occurring at deleterious versus non-deleterious sites and those occurring outside or inside $\mathrm{ROH}$ regions for the given $\mathrm{ROH}$ size. Homozygous non-reference genotypes (1/1) in all sequenced individuals were chosen. Alternate non-reference alleles were classified as deleterious or non-deleterious based on predicted effects as previously described [35]. Congruence with Szpiech et al. [24] was maintained for individual i, across all sites, by denoting $g_{i}^{n, k}$ and $g_{i}^{d, k}$ the total number of sites with $\mathrm{k} \in\{0,1$, $2\}$ alternate alleles at non-deleterious and deleterious sites, respectively. For an individual i, $g_{i, j}^{n, k}$ and $g_{i, j}^{d, k}$ represent the total number of sites with $\mathrm{k} \in\{0,1,2\}$ alternate alleles falling in $\mathrm{ROH}$ class $\mathrm{j} \in\{\mathrm{S}, \mathrm{M}, \mathrm{L}, \mathrm{R}, \mathrm{N}\}$ at non-deleterious and deleterious sites, respectively [24]. Here, S, M, and L indicate the different $\mathrm{ROH}$ length classes (S: small; M: medium; L: long), $\mathrm{R}$ is the union of all three $\mathrm{ROH}$ classes, and $\mathrm{N}$ represents sites located outside any $\mathrm{ROH}$ region [24]. Therefore,

$$
\begin{aligned}
& g_{i, R}^{n, k}=g_{i, S}^{n, k}+g_{i, M}^{n, k}+g_{i, L}^{n, k} \\
& g_{i, R}^{d, k}=g_{i, S}^{d, k}+g_{i, M}^{d, k}+g_{i, L}^{d, k} \\
& g_{i, R}^{n, k}=g_{i}^{n, k}-g_{i, R}^{n, k} \\
& g_{i, N}^{d, k}=g_{i}^{d, k}-g_{i, R}^{d, k}
\end{aligned}
$$

\section{Deleterious and non-deleterious homozygotes in $\mathrm{ROH}$ of any size}

Following Szpiech et al. [24], we compared the proportion of deleterious non-reference homozygotes inside and outside $\mathrm{ROH}$ regions to the corresponding proportion of non-deleterious non-reference homozygotes using the formula:

$$
f_{i, R}^{n}=\frac{g_{i, R}^{n, 2}}{g_{i}^{n, 2}}
$$

where $f_{i, R}^{n}$ is the proportion of non-deleterious $1 / 1$ homozygotes in individual $i$ that fall in any size $R O H$. These proportions of non-deleterious $1 / 1$ homozygotes represent the distribution of non-deleterious homozygotes in $\mathrm{ROH}$ regions. Similarly, we computed

$$
f_{i, R}^{d}=\frac{g_{i, R}^{d, 2}}{g_{i}^{d, 2}}
$$

where $f_{i, R}^{d}$ is the proportion of deleterious $1 / 1$ homozygotes in individual $i$ that fall in any $\mathrm{ROH}$ region [24].
We performed two linear regressions on total genomic $\mathrm{ROH}$ coverage for deleterious and non-deleterious genotypes, and tested statistical significance of results following Szpiech et al. [24]. In addition, we fit a linear model

$$
f_{i, R}=\beta_{0}+\beta_{1} G_{i, R}+\beta_{2} D_{i}+\beta_{3} G_{i, R} D_{i}+\varepsilon
$$

where $f_{i, R}$ is a vector of length 104 containing, for all individuals, the proportion of genome-wide deleterious homozygotes in any $\mathrm{ROH}$ region $\left(f_{i, R}^{d}\right)$ and the proportion of genome-wide non-deleterious homozygotes in any $\mathrm{ROH}$ region $\left(f_{i, R}^{n}\right), G_{i, R}$ is the proportion of the genome covered by $\mathrm{ROH}$ of any size for individual $i$, and $D_{i}$ is an indicator variable with a value of 1 if the observed response is of deleterious homozygotes or a value of 0 for non-deleterious homozygotes [24]. A statistically significant $\beta_{2}$ (via a two-tailed $t$-test) indicates a difference in the intercepts of separate regressions for deleterious and non-deleterious homozygotes, and a statistically significant $\beta_{3}$ (two-tailed $t$-test) indicates a difference in the regression slopes [24].

\section{Deleterious and non-deleterious homozygotes by $\mathrm{ROH}$ size class}

We subsequently tested how deleterious and nondeleterious homozygotes showed increased frequency in different size classes of $\mathrm{ROH}$ regions. It is interesting to explore which $\mathrm{ROH}$ lengths (L, M, or S) exhibited increases in deleterious or non-deleterious homozygotes in the cattle genome. Therefore, we separately evaluated each $\mathrm{ROH}$ size class following Szpiech et al. [24]. Similarly, for homozygous genotypes falling in $\mathrm{ROH}$ of size class $j(j \in\{S, M, L, R, N\})$, we calculated

$$
\begin{gathered}
f_{i, \mathrm{j}}^{d}=\frac{g_{i, \mathrm{j}}^{d, 2}}{g_{i}^{d, 2}} \\
f_{i, \mathrm{j}}^{n}=\frac{g_{i, \mathrm{j}}^{n, 2}}{g_{i}^{n, 2}}
\end{gathered}
$$

for deleterious and non-deleterious $1 / 1$ homozygotes, respectively. We investigated data points for each size class for each individual, using the $f_{i, j}^{d}$ and $f_{i, j}^{n}$ values.

We tested the statistical difference in these regressions with a linear model analogous to the equations from Szpiech et al. [24]. The regression model applied to distinguish deleterious homozygote distributions in $\mathrm{ROH}$ size classes is as follows:

$$
f_{i}^{d}=\beta_{0}+\beta_{1} G_{i}+\beta_{2} C_{i}+\beta_{3} G_{i, R} D_{i}+\varepsilon
$$

where $f_{i}^{d}$ is a vector of length 104 containing, for all individuals, the proportions of genome-wide deleterious homozygotes in large $\mathrm{ROH}\left(f_{i, L}^{d}\right)$, medium $\mathrm{ROH}\left(f_{i, M}^{d}\right)$, and small $\mathrm{ROH}\left(f_{i, S}^{d}\right) . G_{i}$ is the proportion of the genome 
covered by either large $\left(G_{i, L}\right)$, medium $\left(G_{i, M}\right)$, or small $\left(G_{i, S}\right) \mathrm{ROH}$ for individual $\mathrm{i}$, and $\mathrm{D}_{\mathrm{i}}$ is an indicator variable with a value of 1 if the observed response is deleterious homozygotes in large $\mathrm{ROH}$ or a value of 0 if the observed response is deleterious homozygotes in small $\mathrm{ROH}$ (when comparing large and small $\mathrm{ROH}$ ). These comparisons were performed between all possible pairings of $\mathrm{ROH}$ sizes.

\section{Nonsense variants and $\mathrm{ROH}$}

Our study classified homozygotes into two predicted classes: deleterious and non-deleterious. Although the deleterious class exhibited increased variants with deleterious effects, a more informative approach would be to examine a subset of variants with an even higher likelihood of being deleterious, e.g. nonsense mutations as suggested by Szpiech et al. [24], as these are more likely to interfere with normal protein functioning. We tested two sets of predicted nonsense mutations in relationship to their distribution in different $\mathrm{ROH}$ lengths. The first set were predicted as stop gain and stop-loss mutations; the second was a mutation set predicted as frame shift and in-frame mutations, which were classified as loss of function variants. Following Szpiech et al. [24], we divided individuals into two groups: "low- $\mathrm{ROH}$ " and "high- $\mathrm{ROH}$ " individuals to examine nonsense variants in $\mathrm{ROH}$ regions. Individuals with less than $20 \%$ genomic $\mathrm{ROH}$ coverage were classified as low $\mathrm{ROH}$ and those with more than $20 \%$ as high $\mathrm{ROH}$.

\section{Data availability}

Data used in this study are from the 1000 Bull Genome Project (Daetwyler et al. 2014 Nature Genet. 46:858-865). Whole genome sequence data of individual bulls of the 1000 Bull Genomes Project are already available at NCBI using SRA no. SRP039339 (http://www.ncbi.nlm.nih.gov/ bioproject/PRJNA238491).

\section{Additional files}

Additional file 1: Figure S1. Nucleotide diversity distribution on chromosome 23. The X-axis displays the physical position on the chromosome in bp. And the Y-axis shows the corrected SNP number called in $10 \mathrm{kbp}$ bins. Data are from one individual of each breed (HOL, JER, New-RED, and old-RED).

Additional file 2: Figure S2. Heterozygosity distribution as $\log _{2}$ (number of SNPs) per $10 \mathrm{kbp}$ from one individual of each cattle breed (New-RED: blue; HOL: green; JER: red; and Old-RED: orange). The plot only shows bins with heterozygosity greater than 0 .

Additional file 3: Figure S3. $\mathrm{ROH}$ detected from sequence data, HD SNP, and $50 \mathrm{k}$ SNP chip data for chromosome 1 from one Holstein. The X-axis displays the physical position on the chromosome in bp. The Y-axis shows the corrected number of SNPs called in $10 \mathrm{kbp}$ bins. The grey shadows indicate $\mathrm{ROH}$ location called from sequence data, HD SNP, and $50 \mathrm{k}$ SNP chip data.

Additional file 4: Figure S4. Principal component analysis (PCA) depicts the population structure derived from patterns of variability for sequenced individuals from four cattle breeds (HOL: yellow; JER: green; Old-RED: blue; and New-RED: red).
Additional file 5: Figure S5. Three-point ROH statistics for all 104 sequenced individuals. The number of $\mathrm{ROH}$ in the genome of each individual is plotted on the $\mathrm{X}$-axis, the average $\mathrm{ROH}$ size (bp) is depicted on the $\mathrm{Y}$-axis, and nucleotide diversity in a $10 \mathrm{~kb}$ window is shown on the Z-axis (HOL: yellow; JER: green; Old-RED: blue; and New-RED: red).

Additional file 6: Figure S6. The proportion of all genome-wide non-reference homozygotes falling in short $\mathrm{ROH}$ regions versus the genome $\mathrm{ROH}$ coverage for each individual. Red points represent deleterious homozygotes, and orange points represent non-deleterious homozygotes.

Additional file 7: Figure S7. The genome-wide proportion of all non-reference homozygotes falling in $\mathrm{ROH}$ regions versus the genome $\mathrm{ROH}$ coverage for each individual. A: Any $\mathrm{ROH}$ region; $\mathrm{B}$ : Short; C: Medium; and D: Long ROH regions. Red points represent deleterious homozygotes, orange points represent non-deleterious homozygotes, and black points represent synonymous homozygotes.

Additional file 8: Figure S8. The proportion of all genome-wide non-reference homozygotes falling in short $\mathrm{ROH}$ regions versus the genome short $\mathrm{ROH}$ coverage for each individual. Red points represent deleterious homozygotes, orange points represent non-deleterious homozygotes, and black points represent synonymous homozygotes.

Additional file 9: Figure 59. $\mathrm{ROH}$ distribution on chromosome 6. The $\mathrm{X}$-axis plots $\mathrm{ROH}$ on chromosome 6 and the $\mathrm{Y}$-axis represents each of the 104 individuals. Lines indicate $\mathrm{ROH}$ segments across the genome. Red represents HOL, blue JER, brown Old-RED, and purple represents New-RED.

Additional file 10: Table S1. Summary statistics of all sequenced cattle of the Holstein, Jersey, Danish New-red and Danish old-red breeds. The column "Animal" shows the codes for each individual. The $\mathrm{ROH}$ proportion across the genome is shown in column 2. Columns 3, 4, and 5 display the average $\mathrm{ROH}$ size (bp), number of $\mathrm{ROH}$, and total length of $\mathrm{ROH}(\mathrm{bp})$ in the genome, respectively. Nucleotide diversity across the whole genome and outside $\mathrm{ROH}$ are shown in columns 6 and 7. The last three columns display the proportion of short, medium and long $\mathrm{ROH}$.

Additional file 11: Table S2. Genotype counts per cattle individual for sites classified as deleterious.

Additional file 12: Table S3. Genotype counts per individual for sites classified as non-deleterious.

Additional file 13: Table S4. Genotype counts per individual for sites classified as stop gain and stop-loss mutations (nonsense).

Additional file 14: Table S5. Genotype counts per individual for sites classified as frame shift and in-frame mutations (loss of function).

Additional file 15: Figure S10. Signatures of selection based on Fst analysis in sequenced populations. A. Fst values (y-axis) are plotted for each $10 \mathrm{~kb}$ bin for all 29 chromosomes. B. The sharing of $\mathrm{ROH}$ regions in a $10 \mathrm{~kb}$ bin among all individuals for all chromosomes; the $y$-axis is the number of individuals who have the same region of $\mathrm{ROH}$ in a $10 \mathrm{~kb}$ bin. C. Correlation between averaged Fst values (y-axis) and number of individuals sharing the same $\mathrm{ROH}$ regions in a $500 \mathrm{~kb}$ bin (x-axis) (Pearson's product-moment correlation coefficient $=0.20 ; p<0.001)$.

Additional file 16: Figure S11. Signatures of selection based on $\mathrm{iHS}$ testing in sequenced populations. A. The iHS signals ( $y$-axis) between sequenced populations are plotted for all 29 chromosomes. B. Significance of the rSB signals (corresponding $p$ values) for sequenced populations for each chromosome. $\mathrm{C}$. The sharing of $\mathrm{ROH}$ regions in a $10 \mathrm{~kb}$ bin among all individuals for all chromosomes; the $y$-axis is the number of individuals who have the same $\mathrm{ROH}$ region in a $10 \mathrm{~kb}$ bin. D. Correlation between $\mathrm{p}$ values of $\mathrm{iHS}$ signals (y-axis) and number of individuals sharing the same $\mathrm{ROH}$ regions in a $500 \mathrm{~kb}$ bin ( $\mathrm{x}$-axis) (Pearson's product-moment correlation coefficient $=0.22 ; p<0.001$ )

Additional file 17: Figure S12. Permutation of $\mathrm{ROH}$ regions compared with sharing of $\mathrm{ROH}$ regions among individuals. A. Sharing of $\mathrm{ROH}$ regions in a $10 \mathrm{~kb}$ bin among all individuals for all chromosomes; the $\mathrm{y}$-axis is the number of individuals who have the same $\mathrm{ROH}$ region in a $10 \mathrm{~kb}$ bin. $\mathrm{B}$. The randomized $\mathrm{ROH}$ regions over genomes in $10 \mathrm{~kb}$ bins; the $y$-axis is the number of individuals who have the same $\mathrm{ROH}$ region in a $10 \mathrm{~kb}$ bin. 


\section{Abbreviations}

ROH: Runs of homozygosity; HOL: Holstein cattle; JER: Jersey cattle; New-RED: New Danish red cattle; Old-RED: Old Danish red cattle; VEP: Variant Effect Predictor; CNV: Copy number variation; MHC: Bovine major histocompatibility complex; IBD: Identity by descent; PCA: Principal component analysis; LOF: Loss of function.

\section{Competing interests}

The authors declare that they have no competing interests.

\section{Authors' contributions}

QZ developed and planned the study design, coordinated the study, recruited the participants, performed data analyses and drafted the manuscript. BG participated in the study design, analyses of data, and drafting the manuscript. MB contributed analysis tools and participated in drafting the manuscript. MSL participated in study design and drafting the manuscript. GS participated in the study design and drafting the manuscript. All authors read and approved the final manuscript.

\section{Acknowledgement}

Q. Zhang benefited from a joint grant from the European Commission within the framework of the Erasmus-Mundus joint doctorate "EGS-ABG". This research was supported by the Center for Genomic Selection in Animals and Plants (GenSAP) funded by The Danish Council for Strategic Research.

\section{Author details}

${ }^{1}$ Department of Molecular Biology and Genetics, Center for Quantitative Genetics and Genomics, Aarhus University, Tjele DK-8830, Denmark. ${ }^{2}$ Animal Breeding and Genomics Centre, Wageningen UR Livestock Research, Wageningen $6700 \mathrm{AH}$, The Netherlands.

\section{Received: 16 September 2014 Accepted: 23 June 2015 Published online: 22 July 2015}

\section{References}

1. Hayes BJ, Bowman PJ, Chamberlain AJ, Goddard ME. Invited review: Genomic selection in dairy cattle: progress and challenges (vol 92, pg 433, 2009). J Dairy Sci. 2009:92(3):1313-3.

2. Brade W, Brade E. Breeding History of German Holstein Cattle. Ber Landwirtsch 2013, 91(2). http://dx.doi.org/10.12767/buel.v91i2.25.g70.

3. Christensen LG. Cattle-Breeding after 1992. Zuchtungskunde. 1989;61(6):428-39.

4. Ramachandran S, Deshpande O, Roseman CC, Rosenberg NA, Feldman MW, Cavalli-Sforza LL. Support from the relationship of genetic and geographic distance in human populations for a serial founder effect originating in Africa. Proc Natl Acad Sci U S A. 2005;102(44):15942-7.

5. Wright S. Systems of mating. II. The effects of inbreeding on the genetic composition of a population. Genetics. 1921;6(2):124-43.

6. Sorensen AC, Sorensen MK, Berg P. Inbreeding in Danish dairy cattle breeds. J Dairy Sci. 2005;88(5):1865-72.

7. Bjelland DW, Weigel KA, Vukasinovic N, Nkrumah JD. Evaluation of inbreeding depression in Holstein cattle using whole-genome SNP markers and alternative measures of genomic inbreeding. J Dairy Sci. 2013:96(7):4697-706.

8. Nomura T, Honda T, Mukai F. Inbreeding and effective population size of Japanese Black cattle. J Anim Sci. 2001;79(2):366-70.

9. Miglior F, Muir BL, Van Doormaal BJ. Selection indices in Holstein cattle of various countries. J Dairy Sci. 2005:88(3):1255-63.

10. Gonzalez-Recio O, de Maturana EL, Gutierrez JP. Inbreeding depression on female fertility and calving ease in Spanish dairy cattle. J Dairy Sci. 2007;90(12):5744-52.

11. Freyer G, Hernandez-Sanchez J, Cassell BG. A note on inbreeding in dairy cattle breeding. Arch Tierzucht. 2005;48(2):130-7.

12. Ku CS, Naidoo N, Teo SM, Pawitan Y. Regions of homozygosity and their impact on complex diseases and traits. Hum Genet. 2011;129(1):1-15.

13. Miglior F, Szkotnicki B, Burnside EB. Analysis of Levels of Inbreeding and Inbreeding Depression in Jersey Cattle. J Dairy Sci. 1992;75(4):1112-8.

14. Weigel K. Controlling inbreeding in modern dairy breeding programs. Adv Dairy Technol. 2006;18:263-74.

15. Bosse M, Megens HJ, Madsen O, Paudel Y, Frantz LA, Schook LB, et al. Regions of homozygosity in the porcine genome: consequence of demography and the recombination landscape. PLoS Genet. 2012;8(11):e1003100.
16. Pusey A, Wolf M. Inbreeding avoidance in animals. Trends Ecol Evol. 1996:11(5):201-6.

17. Koenig S, Simianer H. Approaches to the management of inbreeding and relationship in the German Holstein dairy cattle population. Livest Sci. 2006;103(1-2):40-53.

18. Margolin S, Bartlett JW. The Influence of Inbreeding Upon the Weight and Size of Dairy Cattle. J Anim Sci. 1945;4(1):3-12.

19. Purfield DC, Berry DP, McParland S, Bradley DG. Runs of homozygosity and population history in cattle. BMC Genet. 2012;13.

20. Lencz T, Lambert C, DeRosse P, Burdick KE, Morgan TV, Kane JM, et al. Runs of homozygosity reveal highly penetrant recessive loci in schizophrenia. Proc Natl Acad Sci U S A. 2007;104(50):19942-7.

21. Nalls MA, Guerreiro RJ, Simon-Sanchez J, Bras JT, Traynor BJ, Gibbs JR, et al. Extended tracts of homozygosity identify novel candidate genes associated with late-onset Alzheimer's disease. Neurogenetics. 2009;10(3):183-90.

22. Charlesworth B, Morgan MT, Charlesworth D. The Effect of Deleterious Mutations on Neutral Molecular Variation. Genetics. 1993;134(4):1289-303.

23. Lohmueller KE, Albrechtsen A, Li YR, Kim SY, Korneliussen T, Vinckenbosch $\mathrm{N}$, et al. Natural Selection Affects Multiple Aspects of Genetic Variation at Putatively Neutral Sites across the Human Genome. PLoS Genetics 2011, 7(10). doi:10.1371/journal.pgen.1002326.

24. Szpiech ZA, Xu JS, Pemberton TJ, Peng WP, Zollner S, Rosenberg NA, et al. Long Runs of Homozygosity Are Enriched for Deleterious Variation. Am J Hum Genet. 2013;93(1):90-102.

25. Loftus RT, MacHugh DE, Bradley DG, Sharp PM, Cunningham P. Evidence for two independent domestications of cattle. Proc Natl Acad Sci U S A 1994;91(7):2757-61.

26. Kim ES, Cole JB, Huson H, Wiggans GR, Van Tassell CP, Crooker BA et al. Effect of Artificial Selection on Runs of Homozygosity in US Holstein Cattle. Plos One 2013, 8(11). doi:10.1371/journal.pone.0080813

27. Leocard S. Selective Sweep and the Size of the Hitchhiking Set. Adv Appl Probab. 2009:41(3):731-64.

28. Pemberton TJ, Absher D, Feldman MW, Myers RM, Rosenberg NA, Li JZ. Genomic patterns of homozygosity in worldwide human populations. Am J Hum Genet. 2012;91(2):275-92.

29. Lohmueller KE, Indap AR, Schmidt S, Boyko AR, Hernandez RD, Hubisz MJ, et al. Proportionally more deleterious genetic variation in European than in African populations. Nature. 2008:451(7181):994-U995.

30. Kirin M, McQuillan R, Franklin CS, Campbell H, McKeigue PM, Wilson JF. Genomic Runs of Homozygosity Record Population History and Consanguinity. Plos One 2010, 5(11). doi:10.1371/journal.pone.0013996

31. Carbone I, Jakobek JL, Ramirez-Prado JH, Horn BW. Recombination, balancing selection and adaptive evolution in the aflatoxin gene cluster of Aspergillus parasiticus. Mol Ecol. 2007;16(20):4401-17.

32. Jansen S, Aigner B, Pausch $H$, Wysocki M, Eck S, Benet-Pages A, et al. Assessment of the genomic variation in a cattle population by re-sequencing of key animals at low to medium coverage. BMC Genomics. 2013;14

33. Zhan BJ, Fadista J, Thomsen B, Hedegaard J, Panitz F, Bendixen C. Global assessment of genomic variation in cattle by genome resequencing and high-throughput genotyping. BMC Genomics. 2011;12.

34. Stothard P, Choi JW, Basu U, Sumner-Thomson JM, Meng Y, Liao XP, et al. Whole genome resequencing of Black Angus and Holstein cattle for SNP and CNV discovery. BMC Genomics. 2011;12

35. McLaren W, Pritchard B, Rios D, Chen YA, Flicek P, Cunningham F. Deriving the consequences of genomic variants with the Ensembl API and SNP Effect Predictor. Bioinformatics. 2010;26(16):2069-70.

36. Velankar S, Dana JM, Jacobsen J, van Ginkel G, Gane PJ, Luo J, et al. SIFTS: Structure Integration with Function, Taxonomy and Sequences resource. Nucleic Acids Res. 2013;41(D1):D483-9

37. Andersen B, Jensen B, Nielsen A, Christensen LG, Liboriussen T. Rød Dansk Malkerace-avlsmæssigt of kulturhistorisk belyst. Danmarks HordbrugsForskning. Denmark. 2003.

38. Andersson L, Lunden A, Sigurdardottir S, Davies CJ, Rask L. Linkage Relationships in the Bovine Mhc Region - High Recombination Frequency between Class-li Subregions. Immunogenetics. 1988:27(4):273-80.

39. Ellis SA, Ballingall KT. Cattle MHC: evolution in action? Immunol Rev. 1999:167:159-68.

40. Kauppi L, Sajantila A, Jeffreys AJ. Recombination hotspots rather than population history dominate linkage disequilibrium in the MHC class I region. Hum Mol Genet. 2003;12(1):33-40. 
41. Kambadur R, Sharma M, Smith TPL, Bass JJ. Mutations in myostatin (GDF8) in double-muscled Belgian blue and Piedmontese cattle. Genome Res. 1997;7(9):910-6.

42. Hoglund JK, Sahana G, Guldbrandtsen B, Lund MS. Validation of associations for female fertility traits in Nordic Holstein, Nordic Red and Jersey dairy cattle. BMC Genetics 2014, 15. doi:10.1186/1471-2156-15-8

43. Zimin AV, Delcher AL, Florea L, Kelley DR, Schatz MC, Puiu D et al. A wholegenome assembly of the domestic cow, Bos taurus. Genome Biol 2009, 10(4). doi:10.1186/gb-2009-10-4-r42

44. Li H, Durbin R. Fast and accurate short read alignment with Burrows-Wheeler transform. Bioinformatics. 2009:25(14):1754-60.

45. Li H, Handsaker B, Wysoker A, Fennell T, Ruan J, Homer N, et al. The Sequence Alignment/Map format and SAMtools. Bioinformatics. 2009;25(16):2078-9.

46. McKenna A, Hanna M, Banks E, Sivachenko A, Cibulskis K, Kernytsky A, et al. The Genome Analysis Toolkit: a MapReduce framework for analyzing next-generation DNA sequencing data. Genome Res. 2010;20:1297-303.

47. Sherry ST, Ward MH, Kholodov M, Baker J, Phan L, Smigielski EM, et al. dbSNP: the NCBI database of genetic variation. Nucleic Acids Res. 2001;29(1):308-11.

48. Sherry ST, Ward MH, Kholodov M, Baker J, Phan L, Smigielski EM, et al. dbSNP: the NCBI database of genetic variation. Nucleic Acids Res. 2000;29(1):38-311

49. Nei M, Li WH. Mathematical-Model for Studying Genetic-Variation in Terms of Restriction Endonucleases. Proc Natl Acad Sci U S A. 1979;76(10):5269-73.

50. Yang JA, Lee SH, Goddard ME, Visscher PM. GCTA: A Tool for Genome-wide Complex Trait Analysis. Am J Hum Genet. 2011;88(1):76-82.

51. Price AL, Patterson NJ, Plenge RM, Weinblatt ME, Shadick NA, Reich D. Principal components analysis corrects for stratification in genome-wide association studies. Nat Genet. 2006;38(8):904-9.

52. Purcell $S$, Neale B, Todd-Brown $K$, Thomas $L$, Ferreira MAR, Bender D, et al. PLINK: A tool set for whole-genome association and population-based linkage analyses. Am J Hum Genet. 2007:81(3):559-75.

53. Weir BS, Cockerham CC. Estimating F-Statistics for the Analysis of Population-Structure. Evolution. 1984;38(6):1358-70.

54. Gautier M, Vitalis R. rehh: an R package to detect footprints of selection in genome-wide SNP data from haplotype structure. Bioinformatics. 2012;28(8):1176-7.

55. Sabeti PC, Reich DE, Higgins JM, Levine HZP, Richter DJ, Schaffner SF, et al. Detecting recent positive selection in the human genome from haplotype structure. Nature. 2002;419(6909):832-7.

56. Tang $K$, Thornton KR, Stoneking M. A new approach for using genome scans to detect recent positive selection in the human genome. PLos Biol. 2007:5(7):1587-602.

57. Zhang Q, Calus M, Guldbrandtsen B, Lund MS, Sahana G. Estimation of inbreeding using pedigree, 50k SNP chip genotypes and full sequence data in three cattle breeds. BMC Genetics, in press. 2015

\section{Submit your next manuscript to BioMed Central and take full advantage of:}

- Convenient online submission

- Thorough peer review

- No space constraints or color figure charges

- Immediate publication on acceptance

- Inclusion in PubMed, CAS, Scopus and Google Scholar

- Research which is freely available for redistribution 